\title{
COMPORTAMENTO DA BETA-CICLODEXTRINA ADICIONADA AO LEITE DE CABRA SUBMETIDO AO PROCESSO DE DESIDRATAÇÃO POR "SPRAY-DRYER"
}

\author{
Adriana C. P. DINIZ, Marilde B. LUIZ , Luciano V. GONZAGA, Marcia M. MEIER, \\ Bruno SZPOGANICZ , Roseane FETT
}

\begin{abstract}
RESUMO
Este trabalho avaliou o comportamento do agente encapsulante $\beta$-ciclodextrina ( $\beta$-CD) adicionado ao leite de cabra submetido ao processo de desidratação por "spray-dryer", através de análise termogravimétrica e de cromatografia gasosa. Após a desidratação, a amostra adicionada de $\beta-C D$ apresentou um rendimento real de $10,59 \%$ com taxa de perda de $0,04 \%$ (em relação ao valor teórico esperado $10,6 \%$ ); enquanto na amostra sem adição do agente encapsulante o rendimento real foi de $9,57 \%$, com taxa de perda de $4,27 \%$ (valor teórico esperado $10 \%$ ). Através da análise termogravimétrica (TGA), verificou-se que são volatilizados $44 \%$ e $21 \%$ dos ácidos comerciais $\mathrm{C}_{\mathrm{s}} \mathrm{e} \mathrm{C}_{\mathrm{i}}$, respectivamente. Os resultados cromatográficos mostraram uma perda de aproximadamente $30 \%$ dos ácidos $C_{a}$ e $20 \%$ dos ácidos $C_{10}$, nas amostras de leite de cabra sem $\beta$-CD em relação às amostras com $\beta$-CD. Tais porcentagens estão de acordo com os valores estimados para os ácidos comerciais. Com base nos parâmetros estudados, podemos inferir que há menor perda dos ácidos graxos $\mathrm{C}_{\mathrm{s}}$ e $\mathrm{C}_{\mathrm{i}}$ na amostra de leite de cabra com $\beta-\mathrm{CD}$, provavelmente devido ao efeito encapsulante, aumentando a estabilidade térmica dos ácidos.
\end{abstract}

Palavras-chave: ciclodextrina; encapsulação; leite de cabra; aroma.

\section{SUMMARY}

BETA-CICLODEXTRIN'S BEHAVIOR ADDED GOAT'S MILK SUBMITTED TO THE "SPRAY-DRYER" DEHYDRATION PROCESS. This work evaluated the effect of the encapsulant agent $\beta$-cyclodextrin $(\beta-C D)$ added the goat milk submitted to the "spray-dryer" dehydration process, through thermogravimetric analysis and gas cromatography. After dehydration, the sample added of $\beta$-CD presented a real yield of $10,59 \%$, with a loss rate of $0,04 \%$ (in relation to the expected theoretical value $10,6 \%$ ); while in the sample without addition of the encapsulant agent the real yield was 9,57\%, with a loss rate of $4,27 \%$ (expected theoretical value $10 \%$ ). It was verified that the volatilization of $44 \%$ and $21 \%$ of the commercial acids $C_{\text {a }}$ and $C_{10}$, respectively, through the thermogravimetric analysis (TGA). The cromatographic results showed losses of approximately $30 \%$ of the $C_{s}$ acids and $20 \%$ of the $C_{i}$ acids, in samples of goat milk without $\beta-C D$, in relation to the samples with $\beta$-CD. These percentages corroborate the values estimated for commercial acids. On the basis of these studied parameters, we can infer that there is a minor loss of the fatty acids $\mathrm{C}_{\mathrm{a}}$ and $\mathrm{C}_{\mathrm{t}}$ in the sample of goat milk with $\beta-C D$, probably due to the encapsulant effect, which increase the thermal stability of the acids.

Keywords: cyclodextrin; encapsulation; goat milk; flavor.

\section{1 - INTRODUÇÃO}

Ciclodextrinas são oligossacarídeos cíclicos, também conhecidas como cicloamiloses, cicloglucanos ou dextrinas de Schardinger, produzidas a partir do amido por ação enzimática [23]. A $\beta$-ciclodextrina ( $\beta-C D)$, formada por sete unidades de $\mathrm{D}(+)$-glicopiranoses unidas entre si através de ligações $\alpha-(1 \rightarrow 4)$, é a mais utilizada na área de alimentos [12]. Quanto ao seu metabolismo, considera-se que ela é digerivel especialmente no intestino grosso, quando é fermentada pela flora bacteriana, em animais experimentais e humanos $[7,26,28]$. A $\beta-C D$ não é tóxica ou genotóxica, mesmo quando ingerida em altas doses (mais de $20 \%$ na dieta) [20, 29].

$\mathrm{O}$ interesse dos pesquisadores pelas ciclodextrinas,

Recebido para publicação em 07/08/2001. Aceito para publicação em 26/04/2005(000699).

Depto. de Ciência e Tecnologia de Alimentos - CCA, Universidade Federal de Santa Catarina. Rod. Admar Gonzaga, 1346. CEP: 88034001, Florianópolis-SC. Telefone/Fax: (48) 334-3726. E-mail: rfett@cca.ufsc.br

Depto. de Quimica, Universidade Federal de Santa Catarina Campus Trindade. CEP: 88040-900, Florianópolis-SC. Telefone: (48) 331-9219.

A quem a correspondência deve ser enviada. reside em sua capacidade de formar complexos com substâncias hidrofóbicas, permitindo a formação de complexos de inclusão com uma grande variedade de substâncias orgânicas. Este processo representa uma das mais avançadas tecnologia de encapsulamento molecular, quando comparado a outros é simples e considerado de baixo custo [11]. Como agente encapsulante de interesse industrial, é particularmente importante na inclusão de compostos responsáveis por aromas em alimentos [3, 27].

$\mathrm{Na}$ área de alimentos, diversas pesquisas são realizadas com a finalidade de aplicar ciclodextrinas na melhoria de produtos, quanto aos aspectos nutricionais, organolépticos e também sensoriais. Estudos têm mostrado diferentes aplicações das ciclodextrinas em sistemas alimentares, como: a) fixação e controle da liberação de aromas $[3,4]$; b) modificação do perfil de sabor e odor pelo mascaramento ou remoção dos aromas indesejáveis [5, 10, 15, 18, 23]; c) estabilização de emulsões [21]; d) proteção de ingredientes ativos da degradação pelo calor, luz e oxidação[17]; e) estabilidade de pigmentos naturais $[6,24]$; entre outros $[16,26]$.

O estudo e aplicação de técnicas que visem a prote- 
ção e conservação de aromas e sabor de alimentos processados, tornam-se particularmente importantes por interferir diretamente na qualidade final do produto $[2,13]$. De acordo com SZEJTLI \& SZENTE [26], os processos comerciais usualmente não são efetivos na diminuição ou eliminação dessas substâncias, pois interferem na qualidade nutricional e/ou sensorial. Assim, os processos de encapsulação de compostos responsáveis por sabor e aroma indesejáveis, próprios do alimento ou desenvolvidos durante o processo industrial que comprometem a qualidade sensorial de alguns alimentos, tornam-se importantes.

O leite de cabra apresenta características peculiares quanto ao sabor e odor; estas podem ser atribuídas principalmente ao alto teor de ácidos graxos de cadeia curta como o ácido cáprico, caprílico e capróico $\left(\mathrm{C}_{\mathrm{t}}, \mathrm{C}_{\mathrm{t}}\right.$ e $\left.\mathrm{C}_{\mathrm{t}}\right)$, quando comparado ao leite de vaca [8]. Estudo realizado por LUIZ \& FETT [19], avaliando a influência da $\beta-C D$ na qualidade sensorial do leite de cabra, demonstrou que $0,4 \%$ deste encapsulante molecular adicionado ao leite de cabra minimizava sensívelmente o sabor caprino, provavelmente em função do processo de encapsulamento dos ácidos graxos de cadeia curta do leite pela $\beta-C D$, formando o complexo de inclusão (ou encapsulação molecular) [22].

Este trabalho tem como objetivo avaliar o efeito da $\beta$-CD na decomposição térmica dos ácidos $C_{\text {e }}$ e $C_{1}$, presentes em amostras de leite de cabra submetidas à secagem por "spray-dryer".

\section{2 - MATERIAL E MÉTODOS}

A $\beta$-CD foi gentilmente cedida por CERESTAR (USA, INC.). O leite de cabra (raça Saanen) foi gentilmente cedido por Laticínios D'Ama Ltda.

\section{1 - Preparação da amostra de leite de cabra $\operatorname{com} \beta-C D$}

Ao leite de cabra pasteurizado $\left(70^{\circ} \mathrm{C}, 30 \mathrm{~min}\right.$.), resfriado foi adicionado $\beta-\mathrm{CD}$, homogeneizado até completa dissolução. A concentração utilizada $0,6 \%$, baseou-se nos dados obtidos por LUIZ e FETT [19], os quais demonstraram a eficiência da $\beta$-CD na minimização do sabor caprino do leite de cabra na concentração de $0,4 \%$; considerando o processo de secagem utilizado no presente estudo, optou-se por utilizar um excesso $50 \%$ em relação a esta concentração.

\section{2 - Solução padrão de ácidos graxos $C_{\text {, }}$ e $C_{1}$ 。}

As soluções padrões dos ácidos graxos $\mathrm{C}_{\mathrm{e}} \mathrm{e} \mathrm{C}_{\mathrm{i}}$ foram preparadas dissolvendo 0,02mg e 0,04mg dos ácidos, respectivamente, em $1 \mathrm{~mL}$ de clorofórmio.

\section{3 - Processo de secagem do leite de cabra}

O processo de secagem foi realizado utilizando o processo industrial de desidratação por "spray dryer", com capacidade de até 200 litros. As amostras foram injeta- das com auxilio de uma bomba de forma a manter a temperatura de entrada entre $180-185^{\circ} \mathrm{C}$ e temperatura de saída entre $80-85^{\circ} \mathrm{C}$. O tempo para cada secagem foi de aproximadamente duas horas. Duas amostras foram submetidas ao processo de secagem: amostra controle (42L de leite de cabra) e amostra teste (42L de leite de cabra com 252 gramas de $\beta-C D)$.

\section{4 - Composição centesimal do leite de cabra em pó}

A composição centesimal (umidade, resíduo mineral, proteínas, lipídios, e carboidratos) nas amostras de leite de cabra em pó, com e sem $\beta-\mathrm{CD}$, foi realizada de acordo com os métodos da A.O.A.C. [1] e INSTITUTO ADOLFO LUTZ [14].

\section{5 - Análise termogravimétrica do ácido graxo $\mathbf{C}_{1}$. e C}

A análise termogravimétrica (TGA) dos ácidos graxos $\mathrm{C}_{1}$ e $\mathrm{C}_{\mathrm{s}}$, foi realizada em um aparelho Shimadzu TGA 50, com taxa de aquecimento de $10^{\circ} \mathrm{C} / \mathrm{min}$ e em atmosfera de nitrogênio. A perda de massa de uma amostra é monitorada em função do tempo e temperatura. Para facilitar a visualização das etapas de decomposição da curva de TGA, utilizou-se a derivada termogravimétrica (DTG ou $\mathrm{dm} / \mathrm{dt})[9]$.

\section{6 - Análise cromatográfica do leite de cabra em pó com e sem adição de $\beta-C D$}

Após a desidratação do leite pelo método de secagem por "spray dryer" as amostras de leite de cabra em pó, com e sem $\beta-C D$, foram submetidas ao processo de extração de ácidos graxos livres utilizando-se o método SANTA-MARIA et al. [25] com algumas adaptações.

Para o processo de extração, utilizou-se $1,6 \mathrm{~g}$ de leite de cabra em pó e $60 \mathrm{~mL}$ de hexano/éter etílico (1:1 v/v). A suspensão foi agitada, filtrada e seu volume foi reduzido à metade por evaporação. Adicionou-se $10 \mathrm{~mL}$ de solução $\mathrm{NaOH}$ (pH 10), com a finalidade de desprotonar os ácidos graxos de cadeia curta $(\mathrm{pKa} \approx 5$ ), solubilizados na fase aquosa. Após filtração, foi acidificada com $\mathrm{HCl}$, promovendo a protonação dos ácidos e redução da solubilidade em meio polar, a extração foi realizada utilizando-se duas alíquotas de $15 \mathrm{~mL}$ de hexano/éter etílico (1:1 v/v). A fase orgânica foi filtrada através de $\mathrm{Na}_{2} \mathrm{SO}_{\text {anidro, para }}$ retirada de umidade. O solvente foi evaporado e o ácido obtido foi dissolvido em $1 \mathrm{~mL}$ de $\mathrm{CHCl}_{\text {s }}$ (para cromatografia).

O extrato contendo os ácidos graxos livres (AGL), extraídos das amostras de leite de cabra em pó com e sem adição de $\beta-C D$, foi analisado por cromatografia gasosa em coluna DB-WAX (análise direta de ácidos), em aparelho CG-17A Shimadzu com detector por ionização em chama. Temperatura do injetor de $200^{\circ} \mathrm{C}$, detector de $260^{\circ} \mathrm{C}$, temperatura inicial de análise de $60^{\circ} \mathrm{C}$ e final $240^{\circ} \mathrm{C}$ taxa de aquecimento $10^{\circ} \mathrm{C} / \mathrm{min}$ e isoterma de $5 \mathrm{mi}-$ 
nutos em $240^{\circ} \mathrm{C}$, a vazão do gás de $2 \mathrm{~mL} / \mathrm{min}$. A identificação dos picos de $\mathrm{C}_{\text {e }}$ e $\mathrm{C}$, foi obtido dissolvendo-se amostras destes ácidos (adquiridos da Fluka) em $\mathrm{CHCl}_{\text {. }}$.

\section{3 - RESULTADOS E DISCUSSÃO}

\section{1 - Processo de secagem por "spray dryer"}

O rendimento real de secagem para a amostra de leite sem adição de $\beta$-CD submetida ao processo de secagem por "spray dryer" foi de $9,57 \%$, com uma taxa de perda de 4,29\% em relação ao valor teórico esperado de $10 \%$. Para a amostra adicionada de $\beta-\mathrm{CD}$, o rendimento real foi de $10,59 \%$, com taxa de perda de $0,045 \%$ em relação ao valor teórico de $10,6 \%$. A diferença entre os valores teóricos deve-se à adição de $\beta$-CD na concentração de $0,6 \%$.

A Tabela 1 apresenta o registro detalhado do processo de secagem por "spray dryer" das amostras de leite de cabra com e sem a adição de $\beta$-CD.

TABELA 1 - Registro do processo industrial de secagem por "spray dryer" das amostras de leite de cabra com e sem $\beta$-CD

\begin{tabular}{lcc}
\hline & Leite de cabra puro & Leite de cabra com $\beta$-CD \\
\hline Variáveis operacionais & & \\
Temperatura entrada $\left({ }^{\circ} \mathrm{C}\right)$ & $180-185$ & $180-185$ \\
Temperatura saída $\left({ }^{\circ} \mathrm{C}\right)$ & $80-85$ & $80-85$ \\
$\quad$ Dados da emulsão & & $\beta-\mathrm{CD}$ \\
\hline Encapsulante & - & 10,60 \\
Teor de sólidos teóricos (\%) & 10,00 & 42 \\
Quantidade emulsão $(\mathrm{kg})$ & 42 & \\
\hline$\quad$ Restitados do processo & & 2 \\
Tempo de secagem (h) & 2 & 4,45 \\
Quantidade produto seco $(\mathrm{Kg})$ & 4,02 & 9,54 \\
Rendimento fórmula $(\%)$ & 9,00 & 10,59 \\
Rendimento real $(\%)$ & 9,57 & 0,045 \\
Taxa de perda $(\%)$ & 4,28 &
\end{tabular}

O maior rendimento observado no processo de secagem para as amostras adicionadas de $\beta-C D$, indica a possivel influência deste encapsulante em diminuir a volatilização ou decomposição térmica de componentes do leite.

\section{2 - Composição química das amostras de leite de cabra}

A análise da composição centesimal (umidade, resíduo mineral, lipídios, proteína total e carboidratos) não demonstrou diferença significativa entre as amostras de leite de cabra em pó com e sem adição de $\beta$-CD . Estes resultados, obtidos a partir da análise de leite de cabra em pó comercial, foram utilizados como parâmetros do processo de secagem realizado no presente trabalho.

TABELA 2 - Valores médios da composição química do leite de cabra desidratado com e sem o agente encapsulante e de uma marca comercial, média de três repetições

\begin{tabular}{ccccccc}
\hline $\begin{array}{c}\text { Leite de } \\
\text { cabra }\end{array}$ & $\begin{array}{c}\text { Proteínas } \\
\mathrm{g} / 100 \mathrm{~g}\end{array}$ & $\begin{array}{c}\text { Lipidios } \\
\mathrm{g} / 100 \mathrm{~g}\end{array}$ & $\begin{array}{c}\text { Carboidratos } \\
\mathrm{g} / 100 \mathrm{~g}\end{array}$ & $\begin{array}{c}\text { Cinzas } \\
\mathrm{g} / 100 \mathrm{~g}\end{array}$ & $\begin{array}{c}\text { Umidade } \\
\mathrm{g} / 100 \mathrm{~g}\end{array}$ & $\begin{array}{c}\mathrm{VCT} \\
\mathrm{Kcal} / 100 \mathrm{~g}\end{array}$ \\
\hline Puro & 22,88 & 30,46 & 34,88 & 5,50 & 6,30 & 505,18 \\
Com $\beta-C D$ & 24,16 & 30,75 & 32,59 & 5,75 & 6,40 & 505,15 \\
Comercial & 24,80 & 32,29 & 33,27 & 6,21 & 3,43 & 523,49 \\
\hline
\end{tabular}

A Tabela 2 mostra os valores médios da composição química do leite de cabra desidratado com e sem o agente encapsulante e de uma marca comercial.

\section{3 - Decomposição térmica dos ácidos $C_{1}$ e C}

As Figuras 1 e 2 mostram os termogramas dos ácidos comerciais $\mathrm{C}_{\mathrm{i}}$ e $\mathrm{C}_{i}$, respectivamente. Verifica-se que em $185^{\circ} \mathrm{C}$ já ocorreu volatilização de aproximadamente $44 \%$ do ácido $\mathrm{C}_{\text {e }}$ e $21 \%$ do ácido $\mathrm{C}_{10}$. Portanto, esses dados indicam que há possibilidade de ocorrer perdas destes ácidos

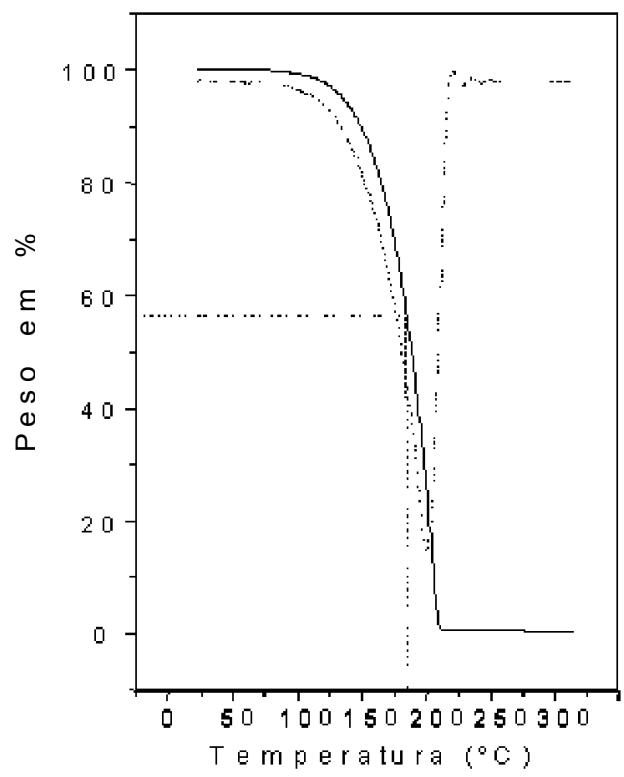

FIGURA 1 - Termograma do ácido C TGA (-), DTG (---)

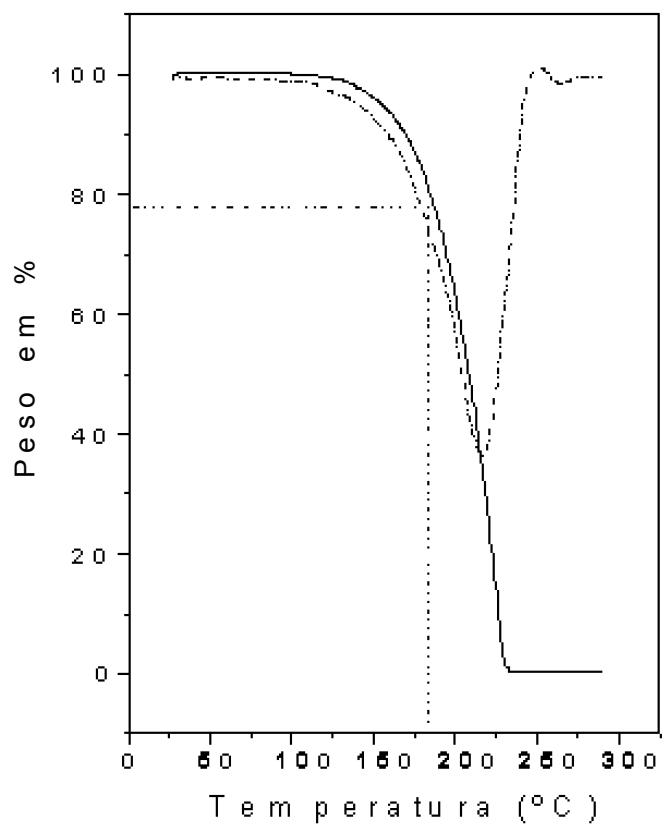

FIGURA 2 - Termograma do ácido $C_{t}$ 。TGA (-), DTG (---) 
durante o processo de secagem das amostras de leite de cabra.

Estudos anteriores, in vitro, mostram que estes ácidos são encapsulados pela $\beta-C D$, sendo que a encapsulação trás um aumento na estabilidade térmica dos ácidos [22].

\section{4 - Análise da decomposição térmica de $C_{\text {, }}$ e $C_{1}$, no leite de cabra}

Os resultados obtidos através de análise cromatográfica das mostras de leite de cabra submetidas ao processo de "spray dryer" estão apresentados na Tabela 3. As porcentagens de perda de $\mathrm{C}_{i} \mathrm{e}_{i 0}$, após o aquecimento a $185^{\circ} \mathrm{C}$, são relativos às amostras adicionadas de $\beta-\mathrm{CD}$ $(100 \%)$.

TABELA 3 - Resultados obtidos a partir de análise cromatográfica do leite de cabra com e sem adição de $\beta$-CD

\begin{tabular}{cccccc}
\hline \multirow{2}{*}{ Amostra } & \multicolumn{2}{c}{ Área do Pico } & & \multicolumn{3}{c}{$\begin{array}{c}\text { Perda após aquecimento } \\
185^{\circ} \mathrm{C}(\%)\end{array}$} \\
\cline { 2 - 3 } \cline { 5 - 6 } \cline { 5 - 6 } & $\mathrm{C}_{8}$ & $\mathrm{C}_{10}$ & & $\mathrm{C}_{8}$ & $\mathrm{C}_{10}$ \\
\hline Sem $\beta-\mathrm{CD}$ & 14.449 & 40.672 & & 30,7 & 20,5 \\
Com $\beta-\mathrm{CD}$ & 20.865 & 51.202 & & - & - \\
\hline
\end{tabular}

Verifica-se que as amostras sem o agente encapsulante apresentam uma perda de $\mathrm{C}_{\mathrm{i}}$ e $\mathrm{C}_{10}$ de aproximadamente $30 \%$ e $20 \%$, respectivamente. Tais valores estão de acordo com os valores previstos pela análise de TGA, através da qual estimou-se uma decomposição de $44 \%$ e $21 \%$ de $\mathrm{C}_{\mathrm{s}}$ e $\mathrm{C}_{\mathrm{i}}$, respectivamente, nas amostras de ácido puro. Os valores de perda dos ácidos no leite são um pouco menores que os valores estimados, principalmente para o ácido $\mathrm{C}_{10}$. Isto se deve, provavelmente, as interações destes ácidos com outras substâncias do leite, aumentando sua estabilidade térmica.

Os resultados de perda de $\mathrm{C}_{10}$ e $\mathrm{C}$, nas amostras sem $\beta-C D$, sugerem que realmente este agente encapsulante impede a decomposição destes componentes do leite. Este aumento na estabilidade térmica dos ácidos se deve à possibilidade de formação dos complexos de inclusão $\beta-C D / C$ e $\beta-C D / C_{1}$. Estes complexos de inclusão já foram estudados in vitro, onde foi confirmada sua maior estabilidade térmica em relação aos ácidos não inclusos [22].

Correlacionando os resultados da análise térmica, perda dos ácidos no leite e o rendimento de secagem das duas amostras de leite de cabra, verifica-se que a $\beta-C D$ foi efetiva na proteção dos ácidos graxos $\mathrm{C}_{\mathrm{s}}$ e $\mathrm{C}_{10}$, quando submetidos à tratamento térmico de $185^{\circ} \mathrm{C}$ no leite de cabra, diminuindo sua perda durante o processo de secagem do leite de cabra por "spray dryer".

\section{4 - CONCLUSÕES}

Correlacionando os resultados do rendimento no processo de desidratação e das análises térmica e cromatocráfica das amostras de leite de cabra com e sem o agente encapsulante, verifica-se que a $\beta$-CD foi efetiva na prote- ção dos ácidos graxos de cadeia curta do leite $\left(\mathrm{C}_{\text {e }}\right.$ e $\left.\mathrm{C}_{\text {。 }}\right)$, quando submetidos a altas temperaturas $\left(180-185^{\circ} \mathrm{C}\right)$, diminuindo suas perdas durante o processo de secagem do leite de cabra por "spray dryer". Os resultados deste trabalho, tomados em conjunto, permitem concluir que a desidratação da amostra de leite de cabra adicionada de $\beta$-CD $(0,6 \%)$, através do processo industrial de secagem por "spray dryer", é viável, não ocorrendo comprometimento dos complexos de inclusão formados. A análise termogravimétrica mostrou ser uma técnica bastante útil na previsão da decomposição dos ácidos.

\section{5 - REFERÊNCIAS BIBLIOGRÁFICAS}

[1] ASSOCIATION OF OFFICIAL ANALYTICAL CHEMISTS. Official methods of analysis of the Association Analytical Chemists. 14 ed.. Washington, D.C., p. 276$298,1984$.

[2] CAMinO, G.; ZANETTI, M. e TROTTA, F. Thermal degradation of ciclodextrins. Polymer degradation and stability., v.69, p.373-379, 2000.

[3] CARDello, H. M. A. B e Celestino, E. M. Encapsulação de aromas e sabores: utilização de amidos como agentes encapsulantes. Bol. SBCTA, v.30, n.2, p.166-171. Jul/dez., 1996.

[4] CHANG, Y.I. e REINECCIUS, G. A Interation of betaciclodextrin with enamtiomers of limonnene and carvone. Journal of Food Science, v.55, n.6, p.1686$1688,1990$.

[5] DINIZ, A.C.P. Avaliação nutricional do leite de cabra adicionado de $\beta$-ciclodextrina. Florianópolis, 2001. 98 p. Dissertação de mestrado, Departamento de Ciência e Tecnologia de Alimentos do Centro de Ciências Agrárias, Universidade Federal de Santa Catarina (UFSC).

[6] FONTANA, J.D.; MENDES, S.V.; PERSIKE, D.S.; PERACETTA, L. e PASSOS, M. Carotenóides: cores atraentes e ação biológica. Capturado em 15 março.

On 1 in e. Dis pon ive 1 na Internet http:/ / www.biotecnologia.com.br/bio/13 e.htm, 2001.

[7] FOGARTY, W.M.; KELLY, C.T. e HAMILTON, L.M. Review: cyclodextrins and their interation with amylolytic enzymes. Enzyme and Microbial Technology, v. 26, p. 561-567, 2000.

[8] HAEnlEin, F.G.W. Producing quality on goat milk. Dairy Goat Journal, v.66, n.5, p.59, 1988.

[9] HAINES, P. J. e WILBURN, F. W. Differential Thermal Analysis and Differencial Scanning Calorimetry. In: HAINES, P. J. Thermal Methods of Analysis. Oxford: Blackie Academic \& Professional, v.63, p. 22, 1995.

[10] HAMILTON, R.M., PARK, L.A. e HEADY, R. E.. Eliminating undesirable taste from coffee and tea extracts and products. US Patent. v.3, p. 528-819, 1970.

[11] HEDGES, A. R., SHIEH, W. J. e SIKORSKI, C. T. Use of cyclodextrins for encapsulation in the use and the treatment for food products. In: Encapsulation and Controled Release of Food Ingredients, 590p., Washington. ACS Symp. Ser. Whashington: Am. Chem. Soc., 1995.

[12] HELENA QI, Z. e HEDGES, A. Use of cyclodextrins for 
flavors. Flavor technology, 1995.

[13] HODGINS, D. e SIMMONDS, D. Sensory technology for flavor analysis. Cereal Food Word1, v.40, n.4, p.186-91, 1995.

[14] INSTituTO ADOLFO LUTZ. Leite...In: Normas analiticas do Inst. Adolfo Lutz, métodos quimicos e físico-químicos para análise de alimentos. 3 ed. São Paulo; v.1, 1985.

[15] ITO, K., KIKUCHI, K., OKAZAKI, N. e KOBAYASHI, S. Retention of aroma components in liquors with cyclodextrins. Agric. Biol. Chem. v.52, n.11, p.27632769, 1988.

[16] KOllengode, A. N. R. e HANNA, M. A. Cyclodextrin complexed flavors retention in extruded starches. Journal of Food Science, v.62, n.5, p.1057-1060, 1997.

[17] LINDEN, G. e LORIENT, D. New ingredients in food processing: biochemistry and agriculture. Book Reviews/Carbohydrate Polymers, v.44, p.273, 2001.

[18] LINDNER, K., SZENTE, L. e SZEJTLI, J. Food flavoring with $\beta$-cyclodextrin-complex flavor substances. Acta Alimentaria, v.10, n.3, p.175-185, 1981.

[19] LUIZ, M.T.B. e FETT, R. Processo de eliminação do "off flavor" em leite de cabra e derivados utilizados. PI 9802188-8. BR Patente. A23C 9/127. 23 jun. 1998.

[20] MARQueS, H. M. C. Cyclodextrins' derivates. Absorption, toxicity, matabolism and fate. Ver. Port. Farm., v.44, n.4, p.147-156, 1994.

[21] McCLEMENTS, D.J. e DECKER, E.A. Lipid oxidation in oil-in-water emulsions: impact of molecular environment on chemical reactions in heterogeneous food systems. J. of Food Science, v.65, n.8, p.1270-1329, 2000.

[22] MEIER, M.M., DRUNKLER, D.A., LUIZ, M. T. B., SZPOGANICZ, B. e FETT, R. The influence of betacyclodextrin on goaty flavour. Characterization of synthetic inclusion complexes with capric acid and caprylic acid. Britsh Food Journal, v.103, n.4, p.281290, 2001.
[23] MUNOZ-Botela, S., DEL CASTillo, B. e MARTiN, M. A.. Las ciclodextrinas. Características y aplicaciones de la formacion del complejo de inclusion. Arsh. Pharm., v.36, n.2, p.187-198, 1995.

[24] PROVENZI, G. Estabilidade de enocianinas adicionadas de $\beta$ e $\gamma$-ciclodextrina e aplicação em yogurte e gelatina. Florianópolis, 2001. $97 \mathrm{p}$. Dissertação de mestrado, Departamento de Ciência e Tecnologia de Alimentos do Centro de Ciências Agrárias, Universidade Federal de Santa Catarina (UFSC).

[25] SANTA-MARIA, G., MARTÍNEZ-CASTRO, I., HIERRO M. T. G., RUIZ-SALA, P. Triglyceride composition of ewe, cow and goat milk fat. J. Am. Oil Chemical Soc., v.73, p.283 293, 1996 .

[26] SZEJTLI, J. e SZENTE, L. Estabilization of flavour by cyclodextrins. In: Flavour encapsulation. Washington: Am. Chem. Soc., cap. 16, p.148-57, 1988.

[27] SZEJTLI, J. e SZENTE, L. Molecular encapsulation of natural and synthetic coffee flavor with b-cyclodextrin. Journal of Food Science, v.51, n.4, p.1024-1027, 1986.

[28] SUZUKI, M. e SATO, A. Nutritional significance of cyclodextrins: indigestibility and hypolipemic effect of $\alpha$ cyclodextrin. Journal Nutrition Science Vitaminol., v.31, p.209-223, 1985.

[29] TOYODA, K., SHODA, T., UNEYAMA, C., TAKADA, K. e TAKAHASHI, M. Food and Chemical Toxicology, v.35, p.331-336, 1997.

\section{6 - AGRADECIMENTOS}

Os autores agradecem à DUAS RODAS INDUSTRIAL Ltda por colocar a disposição o equipamento "Spraydryer" para a realização da secagem das amostras de leite de cabra. LATICÍNIOS D'AMA Ltda. pelo fornecimento do leite de cabra. Cerestar (USA, INC.) pela doação da $\beta$-CD. Ao CNPq pelo auxílio financeiro. 